\title{
Evaluation de l'abondance des kystes des protozoaires flagellés dans les eaux usées exploitées en agriculture maraîchère en zone urbaine : Cas de la ville de Yaoundé (Cameroun)
}

\author{
AJEAGAH Gideon A, Chumtchoua Armel Landry, Mbouombouo Mama, Foto Menbohan Samuel and \\ NJINE Thomas. \\ Laboratory of Hydrobiology and Environment, University of Yaoundé 1 BP337 Yaoundé, Cameroon \\ Correspondant email ; ajeagahg@yahoo.com \\ Original submitted in on 1st September 2016. Published online at www.m.elewa.org on 30th November 2016 \\ http://dx.doi.org/10.4314/jab.v107i1.10
}

\section{RÉSUMÉ}

Objectifs: Evaluer l'abondance des formes de dissémination des protozoaires flagellés dans les eaux usées exploitées en agriculture maraîchère dans certains bas fonds de la ville de Yaoundé.

Méthodologie et résultats: Une étude a été menée de novembre 2013 à avril 2014 avec une phase d'identification et d'analyse bio-statistique de 2015-2016. Des échantillonnages des eaux ont été effectués. Les kystes des protozoaires flagellés ont été identifiés à l'aide de la coloration au Lugol après concentration des échantillons suivant la méthode par flottaison au sulfate de zinc. Les analyses biologiques des eaux révèlent la présence des kystes des Protozoaires flagellés avec des densités moyennes de $101 \pm 70$ kystes/L pour Giardia intestinalis, $34 \pm 20$ kystes/L pour Chilomastix mesnili, $3 \pm 6$ kystes/L pour Retortamonas intestinalis et $1 \pm 3$ kystes/L pour Enteromonas hominis. En général, les plus fortes densités de kystes sont enregistrées pendant la petite saison des pluies (172 $\pm 108 \mathrm{kystes} / \mathrm{L})$. Les analyses statistiques montrent des corrélations significatives $(P \leq 0,05)$ entre la densité des kystes des protozoaires flagelles identifiées et les paramètres physico-chimiques tels que les MES, l'oxydabilité et la turbidité.

Conclusions et application des resultsts: Les plantes doivent être bien lavées, désinfectées et bien cuites avant leurs consommations afin de réduire les risques sanitaires.

Mots clés : kystes, protozoaires flagellés, agriculture maraîchère, bas-fonds et zone urbaine.

\begin{abstract}
Objectives: To evaluate the abundance of environmental forms of flagellated protozoa in waste water that is exploited for urban wetland agricultural practices in Yaoundé.

Methodology and results: A study was carried out from November 2013 to April 2014, with bioenvironmental and bio-statistical assessment from 2015-2016. Sampling took place on monthly basis. The flagellated protozoan cysts were identified by lugol coloration after concentrating the samples according to the method -of flotation using zinc sulphate. Biological analysis of water showed the presence of flagellated protozoan cysts with average densities of (101 \pm 70 cysts/L) for Giardia intestinalis, Chilomastix mesnili (34
\end{abstract}


\pm 20 cysts/L), of Retortamonas intestinalis ( $3 \pm 6$ cysts/L) and of Enteromonas hominis $(1 \pm 3$ cysts/L). Generally, the highest cysts densities were registered during the short rainy season (172 \pm 108 cysts/L). The Statistical analysis showed positive correlations $(P \leq 0,05)$ between the density of these cysts and physic-chemical variables such as suspended solids, oxydability and turbidity. This testifies the importance of colloidal or suspended particles in the transportation of micro-organisms in water.

Conclusion and application of results: In view of these results and the importance of urban agricultural practices in Yaoundé, plants must be well washed and properly cooked before consumption in order to avoid a health risk that is limited to the spread of this waterborne pathogen in this metropoly.

Keys words: Cyst, flagellated protozoa, wetland agricultural, dregs and urban zone.

\section{INTRODUCTION}

L'un des Objectifs du Millénaire pour le Développement $(\mathrm{OMD})$ vise à éliminer l'extrême pauvreté et la faim. Malgré les efforts fournis par les programmes de développement pour atteindre cet objectif, environ 1,2 milliard de personnes vivent toujours dans l'extrême pauvreté à travers le monde (OMD, 2013). Les objectifs de développement durable sont un ensemble d'objectifs portés par l'Organisation des Nations unies et ayant pour but le développement international. Ils remplacent les objectifs du millénaire pour le développement, qui se terminent en 2015, et dont les avancées ont permis une nette évolution. Ces nouveaux objectifs (ODD), portant sur la période 2015-2030, devraient être opérationnels au 1er janvier 2016. Ce "nouveau référentiel du développement du monde" est évalué par divers indicateurs (ODD, 2015).La croissance démographique exponentielle dans les villes suite au phénomène de l'exode rural. La ville de Yaoundé au Cameroun comme l'essentiel des villes des pays en voie de développement (PED) n'échappe pas à cette situation qui est à l'origine d'une insécurité alimentaire et d'un taux de chômage élevé. L'agriculture maraîchère, une activité en plein essor, semble être une alternative intéressante pour résorber une partie du chômage et fournir des aliments frais à la ville (Ndiaye et al., 2010). Pour assurer la pérennité de cette activité en toutes saisons, les populations utilisent l'eau des bas fonds comme moyen d'irrigation. Pourtant, ces cours d'eau constituent des réceptacles terminaux des déchets de toutes sortes (liquide et solide) (Lamizana et al., 2008). Les impacts répétitifs portés aux eaux de surface par les déchets issus des activités anthropiques compromettent sérieusement la qualité de cette ressource qui est malheureusement réutilisée à l'état brut (sans traitement préalable) pour la production des cultures maraîchères (Kouam Kenmogne et al., 2010). Ces eaux usées véhiculent de nombreux microorganismes (Bactéries, Virus, Protozoaires, Helminthes) dont certains sont responsables de plusieurs maladies (Ahmed et al, 2013 ; Cissé et al., 2011). Parmi ces organismes, nous avons les Protozoaires flagellés qui sont des organismes microscopiques appartenant à l'embranchement des Sarcomastigophora, au sous-embranchement des Mastigophora (présence d'organites locomoteurs de type flagelle) et présentant pour certains deux phases dans leur cycle de développement (trophozoïtes et kystiques) (Petithory et al., 1998). Dans l'environnement, ces organismes sont retrouvés sous forme de kystes qui assurent leur dissémination. De tous ces kystes de flagellés, seul le kyste de Giardia intestinalis a un pouvoir pathogène connu (Lacoste, 2009). En effet, l'ingestion de seulement 10 kystes de ce flagellé suffit à infecter l'homme (Adam, 1991 ; Wolfe, 1992). II est responsable de la giardiose qui se manifeste par des diarrhées aiguës, des douleurs abdominales, des nausées et des pertes de poids (Raccurt et al., 2006). L'infection par Giardia intestinalis est la première cause de gastro-entérite parasitaire dans le monde et le nombre de nouveaux cas est estimé à 200 millions par an (Furness et al., 2000). D'après Lacoste (2009), la giardiose est l'affection parasitaire humaine la plus répandue à travers le monde. La contamination par les kystes de protozoaires flagellés constitue un problème de santé publique et doit faire l'objet 
d'une attention particulière (Ajeagah et al., 2007) car ces eaux utilisées pour arroser les cultures hébergeraient des kystes qui peuvent contaminer les produits maraîchers destinés à la consommation. Cependant, les données disponibles sur la distribution des pathogènes flagellés dans les eaux d'irrigation en zone urbaine au Cameroun sont encore au stade élémentaire. Les seules informations relatives aux protozoaires

\section{MATÉRIEL ET MÉTHODES}

Description des sites d'étude et prélèvement des échantillons d'eau : La ville de Yaoundé est située au Sud de la région du Centre entre $3^{\circ} 30^{\prime}$ et $3^{\circ} 58^{\prime}$ de latitude Nord et entre $11^{\circ} 20^{\prime}$ et $11^{\circ} 40^{\prime}$ de longitude Est et à une altitude moyenne de $750 \mathrm{~m}$ (Suchel, 1988). Le réseau hydrographique est principalement constitué par le Mfoundi et ses affluents. Sur la base de bas fonds exploités pour la pratique intensive de l'agriculture maraîchère et l'exploitation des cours d'eau pour l'irrigation des cultures, quatre sites d'étude appartenant au département du Mfoundi et aux les arrondissements de Yaoundé et 4 et 6 ont été choisis. II s'agit d'Ekounou arrosé par le cours d'eau Ekié avec deux stations (Ek1 et Ek2), Mokolo et Nkolbisson arrosés par le cours d'eau Abiergue avec deux stations à Mokolo (Mo1 et Mo2) et deux autres à Nkolbisson (Nk1 et Nk2) et Etoug-Ebe arrosé par le cours d'eau dans les eaux d'irrigation sont celles de Kouam Kenmogne et al. (2010) dans le bassin versant de l'Abiergué (Yaoundé-Cameroun) et de Tsama et al. (2010) dans les boues de vidange à Nomayos (Yaoundé - Cameroun). La présente étude a pour but de rechercher, d'isoler et d'identifier les kystes des protozoaires flagellés dans les eaux utilisées en agriculture maraîchère dans quatre bas fonds de la ville de Yaoundé.

Biyeme avec deux stations (Et1 et Et2). Les stations (Ek1, Et1, Mo1 et Nk1) sont situées sur les cours d'eau tandis que les stations (Ek2, Et2, Mo2 et Nk2) sont des endroits aménagés pour arroser les cultures (Figure 1). Plusieurs campagnes d'échantillonnage ont été effectuées sur les eaux de ces sites. Cette étude s'est déroulée de novembre 2013 à avril 2014 suivant une fréquence mensuelle de prélèvement et les analyse écologique et épidémiologique se sont poursuivies de 2015-2016. Les données obtenues ont été regroupées en saison : GSS (Grande saison sèche) et PSP (Petite saison des pluies). Au total six échantillons ont été analysés à chaque point de prélèvement. Les échantillons ont été prélevés dans des flacons en polyéthylène de $1000 \mathrm{~mL}$ transportés au laboratoire de hydrobiologie et environnement de l'université de Yaoundé I pour analyse. 


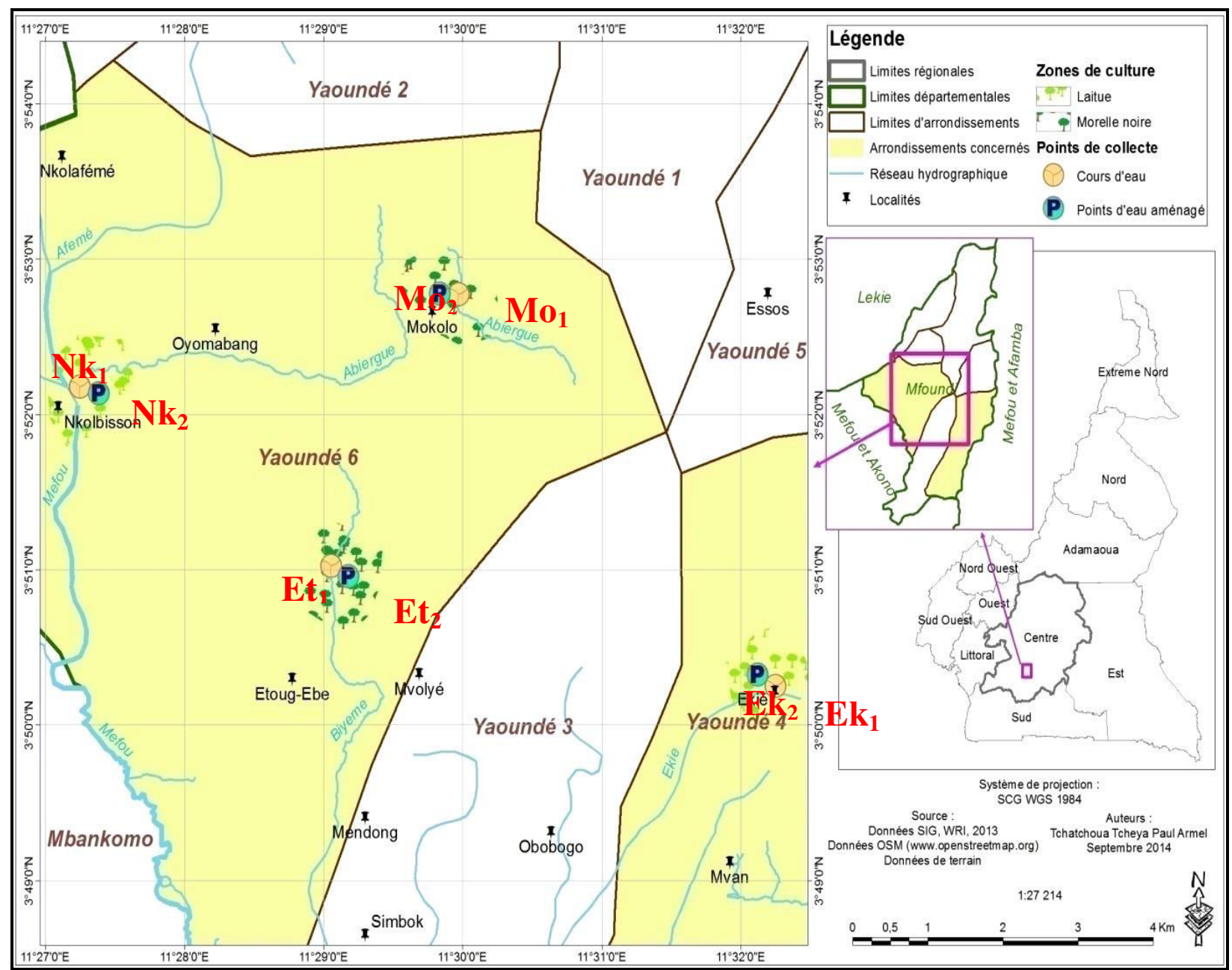

Figure 1 : Carte des stations d'échantillonnage

Analyses physico-chimiques : Les paramètres physico-chimiques examinés ont été la turbidité, les matières en suspension (MES), les nitrates $\left(\mathrm{NO}_{3}{ }^{-}\right)$, les orthophosphates ( $\left.\mathrm{PO}_{4} 3-\right)$ et l'oxydabilité. Les échantillons destinés aux analyses physico-chimiques ont été prélevés dans des flacons de $1000 \mathrm{~mL}$. Les valeurs de l'oxydabilité sont exprimées en $\mathrm{mg} / \mathrm{L}$ de $\mathrm{KMnO}_{4}$ tandis que celles des MES sont exprimées en $\mathrm{mg} / \mathrm{L}$. Les valeurs de la turbidité sont exprimées en unité turbidité formazine (formazine turbidity unity, FTU). Excepté l'oxydabilité qui a été analysée par volumétrie, les autres paramètres ont été déterminés par spectrophotométrie.

Recherche, isolement et identification des kystes des protozoaires flagellés: Pour la recherche et l'isolement des kystes des protozoaires flagellés, on laisse sédimenter $1000 \mathrm{~mL}$ d'échantillon pendant 24 heures. Une fois le culot réparti dans différents tubes à essais, on fixe à l'aide du formaldéhyde (10\%), puis on colore au Lugol simple et on ajoute du sulfate de zinc pour favoriser la flottaison des kystes. L'ensemble est porté dans une centrifugeuse à 500 tours par minutes pendant 5 minutes. Une fois prélevés et posés sur une lame, les kystes sont portés au microscope Olympus pour observation. Les kystes sont identifiés à l'aide des planches de l'OMS (1994).

Analyse des données : Le volume intégral $(V x)$ du culot est enregistré ; après homogénéisation du culot, un volume précis $(\mathrm{Vy})$ est prélevé et réparti dans différents tubes à essais. Le culot final de chaque tube à essais est réparti sur des lames, puis le nombre $(x)$ de kystes contenu dans $1 \mathrm{~L}$ d'échantillon est obtenu par la formule proposée par Ajeagah et al. (2010)

$$
x=\frac{y \cdot V x}{V y}
$$

Avec $V x=$ volume du culot d'1 $\mathrm{L}$ d'échantillon, $V y=$ volume du culot utilisé pour observation, $y=$ nombre de kystes observé dans $\mathrm{Vy}$ 


\section{Ajeagah et al., J. Appl. Biosci. 2016. Evaluation de l'abondance des kystes des protozoaires flagellés dans les eaux usées exploitées en agriculture maraîchère en zone urbaine de Yaoundé (Cameroun)}

Les relations entre la distribution des kystes des parasites et les variables physico-chimiques des eaux

\section{RÉSULTATS ET DISCUSSION}

Les teneurs en MES varient entre $9 \mathrm{mg} / \mathrm{L}$ à la station Ekounou 1 et 15,66 $\mathrm{mg} / \mathrm{L}$ à la station Nkolbisson 1 (Tableau 1). Avec une moyenne de 61,41 $\pm 21,44$ $\mathrm{mg} / \mathrm{L}$, ces valeurs élevées des MES seraient dues aux apports latéraux qui drainent les déchets organiques provenant des rejets divers. Ces valeurs de MES sont supérieures aux normes de la FAO en ce qui concerne les eaux d'irrigation (10 mg/L) (Aghai et Shoukr, 2005). De plus, les tests statistiques montrent des corrélations significatives et positives entre les MES et la turbidité ( $r$ $=0,943 ; p=0,000)$. Selon WHO (2011), la turbidité de l'eau est liée à la présence de la matière organique associée à l'humus et aux particules en suspension dans l'eau. L'augmentation des valeurs de ces paramètres pendant la PSP serait essentiellement liée à la remise en suspension des particules par les eaux des pluies. Auby et al. (1994) affirment en effet que les pluies favorisent l'érosion des particules du bassin versant et leur transport dans les eaux, provoquant ont été évaluées par le test de corrélation de Spearman.

ainsi leur enrichissement en matière organique et leur brassage. Les teneurs en nitrates et en orthophosphates dans les eaux sont relativement faibles et varient entre $0,3 \mathrm{mg} / \mathrm{L}$ de $\mathrm{NO}_{3}$ - à la station $\mathrm{Nk}$ 1 pendant la PSP et $4,3 \mathrm{mg} / \mathrm{L}$ de $\mathrm{NO}_{3}{ }^{-}$à la station Et 2 pendant la GSS pour les nitrates, et entre $0,28 \mathrm{mg} / \mathrm{L}$ de $\mathrm{PO}_{4}{ }^{3-}$ à la station Ek2 pendant la PSP et $1,96 \mathrm{mg} / \mathrm{L}$ de $\mathrm{PO}_{4}{ }^{3-}$ à la station Mo1 pendant la PSP pour les orthophosphates (Tableau 1). Ces valeurs seraient dues à la faible utilisation des engrais azotés et phosphatés dans ces différentes zones pour les cultures d'une part, et à la précipitation des orthophosphates dans les sédiments d'autre part. A cet effet, Djeddi (2007) souligne que les réactions d'adsorption et de précipitation dans les sédiments diminuent les teneurs en orthophosphates dans le milieu. Ces valeurs de nitrates et d'orthophosphates sont inférieures à celles de la FAO pour les eaux d'irrigation (FAO, 2003).

Tableau 1 : Résultats des analyses physicochimiques des échantillons d'eau.

\begin{tabular}{|c|c|c|c|c|c|c|}
\hline Stations & Saison & MES (mg/L) & $\begin{array}{c}\text { Turbidité } \\
\text { (FTU) }\end{array}$ & $\begin{array}{c}\text { Oxydabilité } \\
\left(\mathrm{mg}^{2} \mathrm{~L} \text { de } \mathrm{KMnO}_{4}\right)\end{array}$ & $\begin{array}{c}\mathrm{NO}_{3^{-}} \\
\left(\mathrm{mg} / \mathrm{L} \mathrm{de} \mathrm{NO}_{3}^{-}\right)\end{array}$ & $\begin{array}{c}\mathrm{PO}_{4}^{3-} \\
\left(\mathrm{mg} / \mathrm{L} \text { de } \mathrm{PO}_{4}^{3-}\right)\end{array}$ \\
\hline \multirow[b]{2}{*}{$\mathrm{Ek}_{1}$} & GSS & 46 & 63,66 & 5,79 & 6,9 & 0,87 \\
\hline & PSP & 9 & 24,66 & 6,45 & 1,56 & 0,28 \\
\hline \multirow[b]{2}{*}{$\mathrm{Ek}_{2}$} & GSS & 73 & 128,33 & 8,69 & 0,6 & 0,65 \\
\hline & PSP & 79,33 & 133 & 8,55 & 2,16 & 0,37 \\
\hline \multirow[b]{2}{*}{$E t_{1}$} & GSS & 37,33 & 66,66 & 9,35 & 4,1 & 1,13 \\
\hline & PSP & 30 & 47 & 10,4 & 1,56 & 0,67 \\
\hline \multirow[b]{2}{*}{$\mathrm{Et}_{2}$} & GSS & 70,66 & 100 & 9,87 & 4,3 & 0,86 \\
\hline & PSP & 99,33 & 119 & 10,59 & 2,3 & 0,62 \\
\hline \multirow[b]{2}{*}{$\mathrm{Nk}_{1}$} & GSS & 11,66 & 34,33 & 4,08 & 2,2 & 0,92 \\
\hline & PSP & 8,33 & 33 & 3,16 & 0,3 & 0,31 \\
\hline \multirow[b]{2}{*}{$\mathrm{Nk}_{2}$} & GSS & 61,33 & 81,66 & 1,25 & 1,9 & 0,93 \\
\hline & PSP & 150,66 & 180 & 3,42 & 2,53 & 0,43 \\
\hline \multirow[b]{2}{*}{$\mathrm{Mo}_{1}$} & GSS & 68,66 & 101 & 9,74 & 1,3 & 1,48 \\
\hline & PSP & 104,66 & 135,33 & 18,69 & 1,23 & 1,96 \\
\hline \multirow[b]{2}{*}{$\mathrm{Mo}_{2}$} & GSS & 83,66 & 101 & 6,71 & 2,56 & 1,07 \\
\hline & PSP & 49 & 89,33 & 10,99 & 0,76 & 0,44 \\
\hline
\end{tabular}

Dans les eaux étudiées, l'abondance des kystes des protozoaires flagellés varie entre 0 kyste/L pour Retortamonas intestinalis et Enteromonas hominis et 251 kystes /L pour Giardia intestinalis (Tableau 2). La présence dans ces milieux des kystes des protozoaires flagellés traduisent d'après l'OMS (2012), une contamination fécale des eaux. Ceci rejoint les observations d'Ajeagah et al. (2010) selon lesquelles 
les cours d'eau de Yaoundé sont soumis à la pollution fécale. Selon la FAO (2003), la présence des kystes dans ces milieux rend ces eaux impropres à l'usage agricole. En effet, d'après cette organisation, les eaux destinées à l'usage agricole ne doivent pas contenir de kystes des protozoaires. Sur le plan saisonnier, les densités des kystes varient entre 58 kystes/L à la station Nk 1 pendant la GSS et 310 kystes /L à la station Mo 1 pendant la PSP (Tableau 2). Globalement, les abondances les plus élevées des kystes de protozoaires flagellés sont observées pendant la PSP (Figure2). Ceci pourrait s'expliquer par le fait qu'en saison de pluie, les eaux de ruissellement drainent divers déchets riches en matières organiques provenant des latrines à fond perdu ouvertes pendant cette saison. Ceci expliquerait les corrélations positives obtenues entre les MES, l'oxydabilité et la densité des kystes. En effet, selon Medema et al. (1998), les oocystes et les kystes des parasites sont généralement liés aux matières organiques en suspension dans l'eau. Cette liaison occasionnée par des interactions électrostatiques, celles de Lifshitz-Van der Waals et acides-bases de Lewis (Dai et Boll, 2003) faciliteraient leur dissémination dans le milieu aquatique.

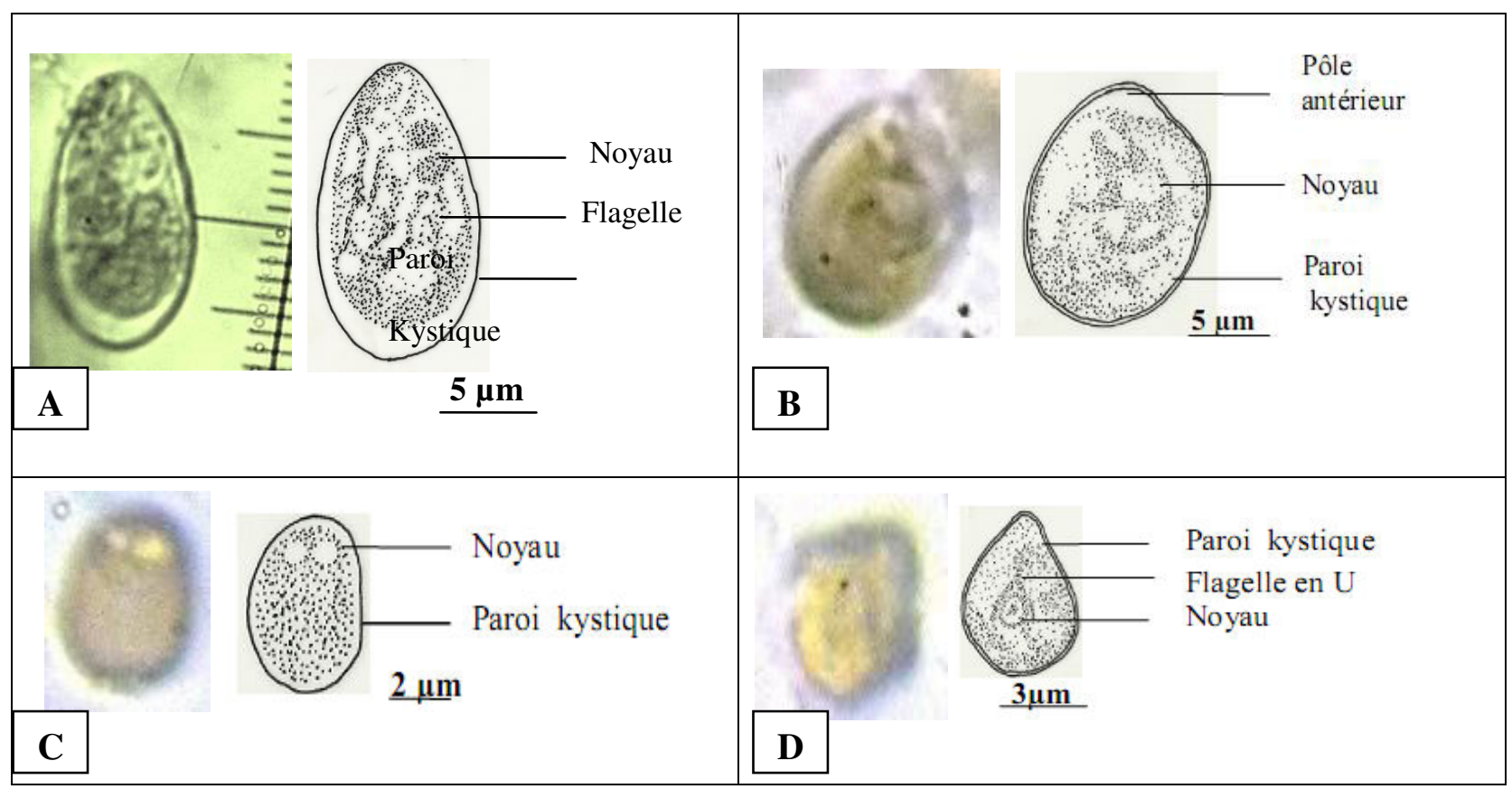

Figure 2: Différents kystes de protozoaires flagellés observés: Giardia intestinalis (A), Chilomastix mesnili (B), Enteromonas hominis (C) et Retortamonas intestinalis (D)

Au cours de cette étude, les analyses biologiques ont permis d'identifier les kystes de quatre espèces des protozoaires flagellés (Figure 2). Le kyste de Giardia intestinalis (figure 2A) a une taille variant entre 8 et 17 $\mu \mathrm{m}$ de long. C'est un kyste ovalaire ou ovoïde avec le contour lisse, assez mince et assez réfringent. Son contenu est fait de deux à quatre noyaux, des flagelles et un axostyle parfois visible qui passe verticalement dans l'axe du kyste. Le kyste de Chilomastix mesnili (figure 2B) a une taille qui varie entre 5 et $10 \mu \mathrm{m}$ de long. II a la forme d'un citron ou d'une poire avec la coque externe lisse, épaisse, très nette et très réfringente. Le contenu est fait d'un gros noyau excentré. Le kyste d'Enteromonas hominis (figure 2C) a une forme ovalaire avec le contour très mince, peu net et peu réfringent. Leur taille varie entre 5 et $7 \mu \mathrm{m}$ de long et leur contenu est fait de deux à quatre noyaux disposés aux extrémités du kyste. Le kyste de Retortamonas intestinalis (figure 2D) à une taille variant entre 4 et $6 \mu \mathrm{m}$ de long. En forme de poire, il est légèrement plus long que large. Son contenu est fait d'un noyau allongé et entouré par un flagelle qui part de l'extrémité rétrécie du kyste et se dédouble en U. 


\section{Ajeagah et al., J. Appl. Biosci. 2016. Evaluation de l'abondance des kystes des protozoaires flagellés dans les eaux usées exploitées en agriculture maraîchère en zone urbaine de Yaoundé (Cameroun)}

Tableau 2 : Résultats des analyses parasitologiques des échantillons d'eau

\begin{tabular}{|c|c|c|c|c|c|c|}
\hline \multirow{2}{*}{ Stations } & Saisons & $\begin{array}{c}\text { Giardia } \\
\text { intestinalis }\end{array}$ & $\begin{array}{c}\text { Chilomastix } \\
\text { mesnili }\end{array}$ & $\begin{array}{c}\text { Retortamonas } \\
\text { intestinalis }\end{array}$ & $\begin{array}{c}\text { Enteromonas } \\
\text { hominis }\end{array}$ & Densité totale(kyste/L) \\
\hline \multirow{2}{*}{$\mathrm{Ek}_{1}$} & $\mathrm{GSS}$ & 76 & 37 & 0 & 7 & 120 \\
\cline { 2 - 7 } & $\mathrm{PSP}$ & 152 & 43 & 0 & 9 & 204 \\
\hline \multirow{2}{*}{$\mathrm{E}_{2}$} & $\mathrm{GSS}$ & 107 & 52 & 3 & 0 & 162 \\
\cline { 2 - 7 } & $\mathrm{PSP}$ & 115 & 36 & 12 & 0 & 71 \\
\hline \multirow{2}{*}{$\mathrm{Et}_{1}$} & $\mathrm{GSS}$ & 40 & 28 & 3 & 0 & 68 \\
\cline { 2 - 7 } & $\mathrm{PSP}$ & 59 & 9 & 0 & 0 & 84 \\
\hline \multirow{2}{*}{$\mathrm{Et}_{2}$} & $\mathrm{GSS}$ & 64 & 20 & 0 & 0 & 189 \\
\cline { 2 - 7 } & $\mathrm{PSP}$ & 153 & 36 & 0 & 0 & 58 \\
\hline \multirow{2}{*}{$\mathrm{Nk}_{1}$} & $\mathrm{GSS}$ & 32 & 21 & 5 & 0 & 96 \\
\cline { 2 - 7 } & $\mathrm{PSP}$ & 77 & 19 & 0 & 0 & 68 \\
\hline \multirow{2}{*}{$\mathrm{Nk}_{2}$} & $\mathrm{GSS}$ & 39 & 29 & 0 & 0 & 159 \\
\cline { 2 - 7 } & $\mathrm{PSP}$ & 128 & 25 & 6 & 0 & 147 \\
\hline \multirow{2}{*}{$\mathrm{Mo}_{1}$} & $\mathrm{GSS}$ & 92 & 39 & 16 & 0 & 310 \\
\cline { 2 - 7 } & $\mathrm{PSP}$ & 251 & 59 & 0 & 0 & 132 \\
\hline \multirow{2}{*}{$\mathrm{Mo}_{2}$} & $\mathrm{GSS}$ & 88 & 39 & 5 & 0 & 192 \\
\cline { 2 - 7 } & $\mathrm{PSP}$ & 137 & 48 & 0 & 7 & \\
\hline
\end{tabular}

Les faibles densités observées pendant la GSS (Figure 3) seraient attribuables à la rareté des eaux de ruissellement pendant cette saison. Les matières organiques stockées dans les bas fonds pendant la saison des pluies sont progressivement transformées en éléments minéraux qui pourraient constituer des agents stressants pour la survie des kystes. A ce propos, Ajeagah et al. (2007) soulignent que des fortes concentrations en éléments minéraux peuvent augmenter l'inactivation des formes de résistances des organismes parasites. Ceci expliquerait les corrélations négatives obtenues entre les orthophosphates, les nitrates et la densité des kystes.

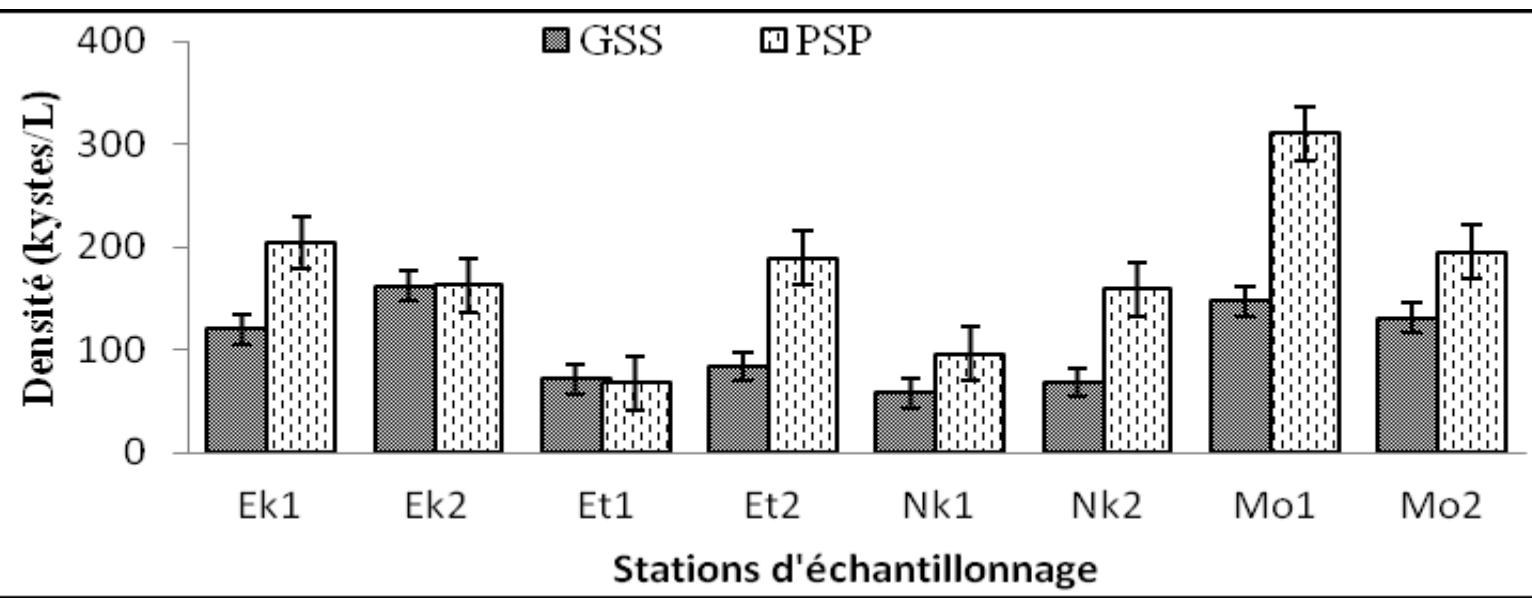

Figure 3: Variation saisonnière des moyennes des densités des kystes des protozoaires flagellés

Sur le plan spatial, les densités élevées des kystes sont obtenues à la station Mo1 avec un total de 372 kystes/L (Figure 4) et pourraient s'expliquer par le fait que les populations déversent dans cette station des déchets de toutes sortes qui sont riches en matières organiques. Cela s'expliquerait également par le fait que cette station est située dans une zone où des latrines à fond perdu et des latrines canons sont vidangées directement dans le cours d'eau. Les faibles densités obtenues à la station Nk1 (423 kystes/L) seraient dues au fait que cette station est un peu plus éloignée des habitations par rapport aux autres. Ainsi, les eaux de cette station auraient donc eu la possibilité de s'auto-épurer par sédimentation et par activité 


\section{Ajeagah et al., J. Appl. Biosci. 2016. Evaluation de l'abondance des kystes des protozoaires flagellés dans les eaux usées exploitées en agriculture maraîchère en zone urbaine de Yaoundé (Cameroun)}

biologique (consommation des kystes par les bactéries et d'autres protozoaires) pendant leur parcours. A cet effet, Bouhoum et al. (1997) affirment que la teneur dans les eaux usées en organismes pathogènes est fortement liée au facteur de développement démographique et socio-économique des populations desservies.

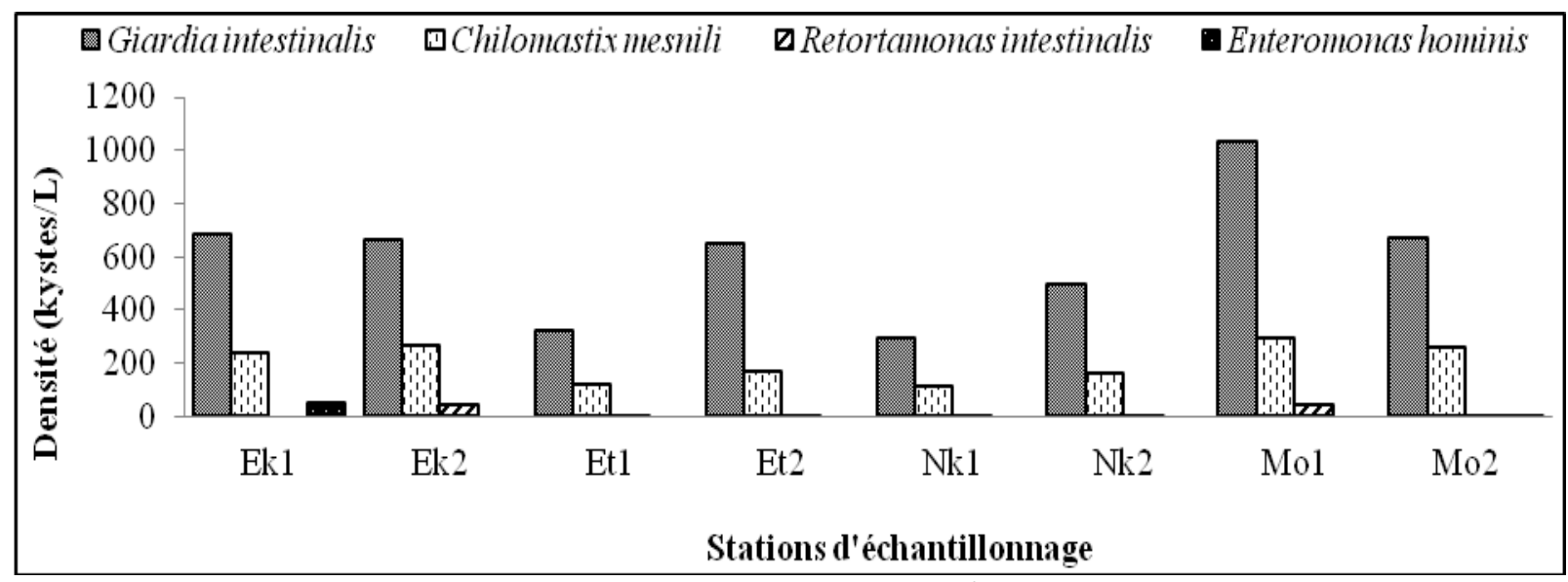

Figure 4 : Variation spatiale des densités des kystes des protozoaires flagellés

\section{CONCLUSION}

L'agriculture est appelée à rester le plus important utilisateur d'eau à l'échelle mondiale dans les décennies à venir. C'est à partir de la révolution verte que l'agriculture a commencé à s'approprier des quantités d'eau de plus en plus importantes. Cette étude a permis l'isolement et la mise en évidence des kystes de protozoaires flagellés dans les eaux exploitées pour les cultures maraîchères à Yaoundé (quartiers Ekounou, Etoug-Ebe, Mokolo et Nkolbisson).Des mesures environnementales appropriées ainsi que les procédés adéquats de traitement de ces eaux s'imposent afin d'éviter un risque sanitaire de grande envergure dans la région. Les plantes arrosées par ces eaux doivent être bien lavées et bien cuites avant leur consommation. La gestion de l'eau en agriculture devra encore mieux

\section{RÉFÉRENCES}

Adam R. D. 1991. The biology of Giardia sp. Microbiology Review, 55: 706-732.

Aghai O. \& Choukr-Allah R. 2005. Impact de l'irrigation par les eaux uses épurées sur la productivité d'une culture de tomate de plein champ, la santé du sol et le bilan d'azote. The use of non conventionna water resources. Bari: CHEAM/EU DG Research, 66 (11) : 165-171.

Ajeagah A. G., Njiné T., Nola T., Foto M. S. \& Wouafo N. M. 2007. Evaluation de l'abondance des formes de résistance de deux s'intégrer avec le traitement de l'eau dans les ménages et les industries avant tout déversement dans la nature. Pour éviter tout risque de "contamination", les canalisations de distribution d'eaux usées traitées doivent être distinctes du réseau de distribution d'eau potable. L'exploitant des eaux d'irrigation doit mettre en place un programme de surveillance des eaux traitées et des sols, qui comporte le suivi analytique des protozoaires flagellés pendant la totalité de la saison d'irrigation. L'installation des toilettes, l'utilisation de l'eau de bon qualité, l'application de génie sanitaire pour réduit le taux de péril fécale sont les mesures les plus importantes pour assurer la diminution des cas de diarrhée par des nids à polluants par des pathogènes (bactéries, virus, protozoaires, helminthes), matières organiques, substances chimiques.

protozoaires pathogènes (Giardia sp. et Cryptosporidium sp.) dans deux biotopes aquatiques de Yaoundé (Cameroun).Cahier d'Etudes et de Recherche Francoa phones/Santé, 17 (3) : 167-172.

Ajeagah G. A., Njine T., Bilong Bilong C. F., Foto S.M. ; Wouafo N. M. , Nola M. , Di G. G. D. \& Huw S. 2010. Seasonal Distribution of Enteric Opportunistic Cryptosporidium Spp.Oocysts and Giardia Spp. Cysts in a tropical water basin, Cameroon. Water, 2: 44-57. 
Ahmed W., Sritharan T., Palmer A., Sidhu J. P. S. \& Tozea S. 2013. Evaluation of bovine fecesassociated microbial source tracking markers and their correlations with fecal indicators and zoonotic pathogens in a brisbane, Australia, Reservoir. Applied and Environmental Microbiology, 79(8): 26822691

Auby I., Manaud F., Mauder D. \& Trut G. 1994. Etude de la prolifération des Algues vertes dans le bassin versant d'Arcachon, IfremerCemagre Ssa-sabarc. 192 p.

Bouhoum K., Mahmid O. Habba K. H., \& Schwartzbrod. 1997. Devenir des oeufs d'helminthes et des kystes de protozoaires dans un canal à ciel ouvert alimenté par les eaux usées de Marrakech. Revue des Sciences de l'Eau, 2 : 217- 232.

Cissé M., N'guessan F., Karamoko Y., Tigoli K., Dje Bi Dje F. \& Gouréne G. 2011. Charge parasitaire des eaux usées du collecteur «Gouro » traversant les communes d'Abobo, Adjamé et Cocody (District d'Abidjan). Les technologies de laboratoire, 6 (25): 96-105.

Dai X. \& Boll J. 2003. Evaluation of attachment the of Cryptosporidium parvum and Giardia lamblia to soil particles. Journal of Environnemental Quality., 32: 296-304.

Djeddi H. 2007. Utilisation des eaux d'une station d'épuration pour l'irrigation des essences forestières urbaines. Mémoire : Université Mentouri Constantine (Algérie), 136p.

FAO (Organisation des Nations Unies pour l'alimentation et l'agriculture). 2003. L'irrigation avec des eaux usées traitées : Manuel d'utilisation. FAO Irrigation and Drainage, $65 p$.

Furness B. W., Beach M. J. \& Roberts J. M. 2000. Giardiasis surveillance. United States, 19921997. Mor mortal Wkly Rep CDC, 49 (7): 113.

Kouam Kenmogne G., Rosillon F., Mpakam H. G. \& Nono A. 2010. Enjeux sanitaires, socioéconomiques et environnementaux liés à la réutilisation des eaux usées dans le maraîchage urbain: cas du bassin versant de l'Abiergué (Yaoundé-Cameroun), VertigO - la revue électronique en sciences de l'environnement, 10 (2) : 1-23.
Lacoste R. 2009. Les parasites intestinaux chez le macaque crabier (Macaca fascicularis): Etude expérimentale et recommandations pour la diagnose et la gestion des Rhizoflagelles et des ciliés. Thèse pour le Doctorat vétérinaire, Faculté de Médecine de Créteil/Ecole Nationale Vétérinaire d'Alfor, 237p.

Lamizana D., Birguy M., Kenfack S. \& Millogo R. J. 2008. Evaluation de la qualité physicochimique de l'eau d'un cours d'eau temporaire du Burkina Faso: Le cas du Massili dans le Kadiogo. Sud Sciences et Technologies, 16: 23-28.

Medema G. J., Schets F. M., Teunis P. F. M. \& Havelaar N. H. 1998. Sedimentation of free and attached Cryptosporidium oocysts and Giardia in water. Appleid Environnemental Microbiology, 64 : 4460-4466.

Ndiaye M. L., Pfeifer H. R., Niang S., Dieng Y., Tonolla M. \& Peduzzi R. 2010. Impacts de l'utilisation des eaux polluées en agriculture urbaine sur la qualité de la nappe de Dakar (Sénégal), VertigO - la revue électronique en sciences de l'environnement, 10(2) : 1-20.

ODD,2015, De nouveaux objectifs développement durable pour " transformer le monde », sur Actu-Environnement, 24 septembre 2015

OMD (Objectifs du Millénaire pour le Développement), 2013. Rapport de Publié par les nations unies, 63p.

OMS (Organisation mondiale de la Santé). 1994. Planches pour le diagnostic des parasites intestinaux. Genève, $29 \mathrm{p}$.

OMS (Organisation mondiale de la Santé). 2012. Directives OMS pour l'utilisation sans risque des eaux usées, des excréta et des eaux ménagères. Utilisation des eaux usées et des excréta en aquaculture, Genève, $63 p$.

Petithory J.C., Ardoin-Guidon F. \& Chaumeil C. 1998. Cahier de formation - Biologie médicale ; Amibes et flagellés intestinaux: amibes oculaires, leur diagnostic microscopique, 11: $237 p$

Raccurt CP, Pannier SC, Eyma E, Verdier RI, Totet A \& Pape JW. 2006 Enteric parasites and AIDS in Haiti. Utility of detection and treatment of parasites in family members. Médecine Tropicale, 66 (4): 461.

Suchel J.B. 1988. Les climats du Cameroun. Thèse de doctorat d'état. $1177 p$. 
Wolfe M. S. 1992. Giardiasis. Clinical Microbiology Review, 5 (1): 93-100.

WHO (World Health Organization). 2011. Guidelines for drinking-water quality. $4^{\text {th }}$ edition, Geneva, 564 P.

Tsama V., Pial A. C., Youmbi G. T. \& Akoa A. 2010. Incidence de la charge en œufs d'helminthes contenue dans les boues de vidange sur la qualité parasitaire de quelques cultures maraîchères à Nomayos (Yaoundé Cameroun). Afrique Science, 6 (1): 106 115. 